\title{
Response to Invited Commentary
}

\section{Rasmussen-Torvik et al. Respond to "The Perfect Measure of Diastolic Dysfunction"}

\author{
Laura J. Rasmussen-Torvik*, Laura A. Colangelo, Joao A. C. Lima, David R. Jacobs, Jr., \\ Carlos J. Rodriguez, Samuel S. Gidding, Donald M. Lloyd-Jones, and Sanjiv J. Shah
}

\author{
* Correspondence to Dr. Laura J. Rasmussen-Torvik, Department of Preventive Medicine, Feinberg School of Medicine, Northwestern \\ University, 680 N. Lake Shore Drive, Suite 1400, Chicago, IL 60611 (e-mail: ljrtorvik@ northwestern.edu).
}

Initially submitted November 17, 2016; accepted for publication November 22, 2016.

We thank Drs. Pandey and Berry for providing insightful commentary (1) on our study (2), which demonstrated poor overlap among currently used definitions of diastolic dysfunction (DD) in a middle-aged cohort. Pandey and Berry caution against a strategy of trying to find "perfect" echocardiographic criteria for DD and urge that investigators consider the incorporation of blood-based markers and exercise capacity into the definition of DD (1), as these markers can help place echocardiographic measurements into proper context from a clinical point of view.

We agree with Pandey and Berry about the importance of not fixating on a "perfect" definition of DD. Even an imperfect definition of DD could still be a critically important phenotype clinically if it were found to be a strong risk factor for heart failure with preserved ejection fraction (HFpEF) and amenable to treatment for the prevention or delay of HFpEF development. It may be that one of the existing definitions of DD examined in our paper (3-5) is highly predictive of future HFpEF or that incorporation of blood markers, exercise capacity, or another variable will be required to achieve good future HFpEF prediction. In the Multi-Ethnic Study of Atherosclerosis, for example, we are currently performing dynamic echocardiography to study whether the addition of left atrial strain (measured by speckletracking analysis) at rest and during a passive leg-raise maneuver augments echocardiographic definitions of DD. However, until such time as studies demonstrating which DD phenotypes are predictive of future $\mathrm{HFpEF}$ can be completed, we urge investigators to be cognizant of the limitations of the current definitions and understand that they are not interchangeable. For those proposing new definitions of DD, we encourage definitions that are easy to implement in large population studies, as these are the studies needed to establish associations between DD in middle age and subsequent development of HFpEF.

\section{ACKNOWLEDGMENTS}

Author affiliations: Department of Preventive Medicine, Feinberg School of Medicine, Northwestern University,
Chicago, Illinois (Laura J. Rasmussen-Torvik, Laura A. Colangelo, Donald M. Lloyd-Jones); Department of Medicine, School of Medicine, Johns Hopkins University, Baltimore, Maryland (Joao A. C. Lima); Division of Epidemiology and Community Health, School of Public Health, University of Minnesota, Minneapolis, Minnesota (David R. Jacobs, Jr.); Department of Medicine and Public Health Sciences, School of Medicine, Wake Forest University, Winston-Salem, North Carolina (Carlos J.

Rodriguez); A. I. DuPont Hospital for Children, Wilmington, Delaware (Samuel S. Gidding); and Division of Cardiology, Department of Medicine, Feinberg School of Medicine, Northwestern University, Chicago, Illinois

(Sanjiv J. Shah, Donald M. Lloyd-Jones).

This work was supported by contracts HHSN268201300025C, HHSN268201300026C, HHSN268201300027C, HHSN268201300028C, HHSN268201300029C, and HHSN268200900041C from the National Heart, Lung, and Blood Institute (NHLBI), the Intramural Research Program of the National Institute on Aging (NIA), and an intraagency agreement between the NIA and the NHLBI (agreement AG0005), all of which fund the Coronary Artery Risk Development in Young Adults Study.

Conflict of interest: none declared.

\section{REFERENCES}

1. Pandey A, Berry JD. Invited commentary: searching for the perfect measure of diastolic dysfunction-a futile exercise? Am J Epidemiol. 2017;185(12):1228-1230.

2. Rasmussen-Torvik LJ, Colangelo LA, Lima JAC, et al. Prevalence and predictors of diastolic dysfunction according to different classification criteria: the Coronary Artery Risk Development in Young in Adults Study. Am J Epidemiol. 2017; 185(12):1221-1227. 
3. Solomon SD, Janardhanan R, Verma A, et al. Effect of angiotensin receptor blockade and antihypertensive drugs on diastolic function in patients with hypertension and diastolic dysfunction: a randomised trial. Lancet. 2007;369(9579): 2079-2087.

4. Nagueh SF, Appleton CP, Gillebert TC, et al.

Recommendations for the evaluation of left ventricular diastolic function by echocardiography. Eur J Echocardiogr. 2009;10(2): 165-193.

5. Redfield MM, Jacobsen SJ, Burnett JC Jr, et al. Burden of systolic and diastolic ventricular dysfunction in the community: appreciating the scope of the heart failure epidemic. JAMA. 2003;289(2):194-202. 